\title{
Asymmetric Variance Reduction for Pricing American Options
}

\author{
Jean-Pierre Fouque ${ }^{1}$
}

Department of Statistics and Applied Probability, University of California, Santa Barbara, CA 93106-3110, USA

E-mail: fouque@pstat.ucsb.edu

Chuan-Hsiang $\operatorname{Han}^{2}$

Department of Quantitative Finance, National Tsing-Hua University, Hsinchu,Taiwan 30013, ROC.

E-mail: chhan@mx.nthu.edu.tw

\begin{abstract}
Based on the dual formulation by Rogers (2002), Monte Carlo algorithms to estimate the high-biased and low-biased estimates for American option prices are proposed. Bounds for pricing errors and the variance of biased estimators are shown to be dependent on hedging martingales. These martingales are applied to (1) simultaneously reduce the error bound and the variance of the high-biased estimator, and (2) reduce the variance of the low-biased estimator while preserving its biased level. For a class of stochastic volatility models, projected hedging martingales are constructed based on an application of asymptotic expansion of option prices. These martingales are easy to compute. Numerical results demonstrate the robustness and effectiveness of these projected hedging martingales.
\end{abstract}

\section{Introduction}

The right to early exercise a contingent claim is an important feature for derivative trading. An American option offers its holder, not the seller, the right but not the obligation to exercise the contract at any time prior to maturity during its contract life time. Based on the no arbitrage argument, the American option price at time 0 , denoted by $P_{0}$, with maturity $T<\infty$ is considered as an optimal stopping problem [10]. That is, under the risk-neutral probability space $\left(\Omega, \mathcal{F}, \mathbb{P}^{\star},\left(\mathcal{F}_{t}\right)_{t \in[0, T]}\right)$,

$$
P_{0}=\sup _{0 \leq \tau \leq T} \mathbb{E}^{\star}\left\{Z_{\tau} \mid \mathcal{F}_{0}\right\}
$$

where the supremum is taken over all the stopping times $\tau$ bounded by $T$, the discounted payoff is denoted by $Z_{t}=D_{t} \tilde{Z}_{t}$ in which $D_{t}$ is the discount factor and $\tilde{Z}_{t}$ is the payoff at time $t$, both $\left(\mathcal{F}_{t}\right)$ adapted. We assume that $Z_{t}$ satisfies the uniformly integrable condition $\sup _{0 \leq t \leq T}\left|Z_{t}\right| \in L^{p}$ for some $p>1$, and $Z$ is right continuous.

During the last decade, methods of Monte Carlo simulations have made a great progress in solving the American option pricing problem. Among these, primal methods and dual methods provide upper solutions and lower solutions for the American option price respectively. Primal methods such as in [8] and [11] address the optimal stopping problem (1) by approximating the free boundary or the optimal stopping rule; while dual methods such as in [7] and [10] address a stochastic minimization

\footnotetext{
${ }^{1}$ Work supported by NSF grant DMS-0455982.

${ }^{2}$ This work is supported by NSC grant 95-2115-M-007-017-MY2, Taiwan. CH Han is grateful for discussions with Professor Sheunn-Jhi Sheu at the Institute for Mathematics, Academia Sinica, Taiwan.
} 
problem by approximating the optimal supermartingale or martingale respectively. As a result, the primal method induces a low-biased estimate and the dual method induces a high-biased estimate for the American option price. Results from these two methods are useful for practical trading activities: the option seller is typically interested in the high-biased estimate as the hedging strategy is related to (super)martingale; while the option holder is interested in low-biased estimate as the time to early exercise can be simulated.

Based on Rogers' dual formulation [10] this paper proposes and analyzes methods to compute highbiased estimates and low-biased estimates for the American option price. The low-biased estimator is naturally equipped with a variance reduction feature. Primal and dual representations for the American option price by martingales are characterized. The price gap and variance of biased estimators are sensitive to zero-centered martingales. Because the martingales are associated with hedging strategies, we refer them as hedging martingales. It remains a task to search for hedging martingales.

There exists an enormous literature about American option price approximations, which are typical in closed-form or analytic form. Unfortunately the process obtained from an approximate discounted price may not be a martingale or a supermartingale. For instance, in an example given in Lemma 7 in the Appendix, we find that the discounted quadratic approximation in [1] does not posses the (super) martingale property. However, if the delta of an option price approximation is easy to compute, then the corresponding stochastic integral-type martingale becomes easy to construct. This can be seen as one advantage. Another advantage is that the integral-type martingale represents a continuous-time trading activity of dynamic hedge. Therefore the variance of the biased-price estimator represents the quadratic measure of associated hedging errors. In this paper we consider hedging martingales being in stochastic integral type, rather than a discounted approximate price as in [10].

For Monte Carlo simulations, variance reduction methods are important to improve the precision of estimates. In our formulation the low-biased estimator comprises the sample mean of a discounted payoff less a hedging martingale at a stopping time. Hence, based on the optional sampling theorem, the hedging martingale is a natural candidate to play the role of a linear control to reduce the variance of low-biased estimators. For the high-biased estimator of the American option price, the hedging martingale can be understood as a nonlinear control. Theorem 3 in this paper guarantees that a hedging martingale inducing a smaller high-biased estimate will induce a smaller variance, at least in a neighborhood of the optimal hedging martingale. In other words, for high biased estimates, one can reduce the bias and the variance of the estimator at the same time. This effect contradicts typical variance reduction methods for unbiased estimators as used for low-biased estimates. That is, given a stopping rule the hedging martingale will only affect the variance without changing the mean level. This asymmetric variance reduction effect produced by hedging martingales to estimate upper and lower solutions can be observed numerically in Section 3 under Black-Schole models and Section 4 under stochastic volatility models.

The organization of the paper is as follows. In Section 2 the high-biased and low-biased estimates for the American option price are proposed. We deduce representations for primal and dual formulations. The asymmetric behavior between the price bias and the variance are analyzed. In Section 3 the Black-Scholes model is considered. A characterization of the optimal stopping time is obtained. Two dual formulations: (1) hedging martingale by Rogers [10] and (2) hedging supermartingale by Haugh and Kogan [7] are shown to be equivalent. Numerical results to estimate American option prices are demonstrated. In Section 4 multiscale stochastic volatility models are considered. Based on an asymptotic expansion for the option price as in [5], the construction of a projected hedging martingale is proposed and some numerical examples are demonstrated. We conclude this paper in Section 5. 


\section{Primal and Dual Formulations of American Option Prices}

Rogers [10] obtained a dual formulation for the American option problem (1) by solving an inf-sup problem over martingales:

$$
P_{0}=\inf _{M \in H_{0}^{1}} \mathbb{E}^{\star}\left\{\sup _{0 \leq t \leq T}\left(Z_{t}-M_{t}\right) \mid \mathcal{F}_{0}\right\}
$$

where the space of martingales is

$$
H_{0}^{1}=\left\{\left(M_{t}\right)_{0 \leq t \leq T}: \text { martingales with } \sup _{0 \leq t \leq T}\left|M_{t}\right| \in L^{1} \text { and } M_{0}=0\right\} .
$$

The proof is based on the Doob-Meyer decomposition of a super-martingale process, and, in fact, the infimum in (2) is attained so that

$$
P_{0}=\mathbb{E}^{\star}\left\{\sup _{0 \leq t \leq T}\left(Z_{t}-M_{t}^{*}\right) \mid \mathcal{F}_{0}\right\}
$$

where the optimal martingale $M^{*}$ is the unique martingale obtained from the Doob-Meyer decomposition. The following result shows that the American option price is bounded above by a lookback-style option price based on the dual approach and bounded below by a barrier-style option price based on the primal approach.

\subsection{High-biased and Low-biased Estimates}

Proposition 1 Given an integrable martingale $M \in H_{0}^{1}$, and a stopping time $0 \leq \tau \leq T$, the highbiased estimate and the low-biased estimate of the American option price are obtained:

$$
\mathbb{E}^{\star}\left\{Z_{\tau}-M_{\tau} \mid \mathcal{F}_{0}\right\} \leq P_{0} \leq \mathbb{E}^{\star}\left\{\sup _{0 \leq t \leq T}\left(Z_{t}-M_{t}\right) \mid \mathcal{F}_{0}\right\} .
$$

Proof:

From (2), it is easy to obtain an upper-bound solution or a high-biased estimate of the American option price

$$
P_{0} \leq \mathbb{E}^{\star}\left\{\sup _{0 \leq t \leq T}\left(Z_{t}-M_{t}\right) \mid \mathcal{F}_{0}\right\}
$$

for any given integrable martingale $M \in H_{0}^{1}$. On the other hand, for any bounded stopping time $0 \leq \tau \leq T$, it is readily seen that

$$
Z_{\tau}-M_{\tau} \leq \sup _{0 \leq t \leq T}\left(Z_{t}-M_{t}\right)
$$

such that after taking an expectation, the left hand side is equal to

$$
\mathbb{E}^{\star}\left\{Z_{\tau}-M_{\tau} \mid \mathcal{F}_{0}\right\}=\mathbb{E}^{\star}\left\{Z_{\tau} \mid \mathcal{F}_{0}\right\}=\mathbb{E}^{\star}\left\{Z_{\tau}-\tilde{M}_{\tau} \mid \mathcal{F}_{0}\right\}
$$

for any other integrable martingale $\tilde{M} \in H_{0}^{1}$ due to the optional sampling theorem. Therefore a lower bound solution or a low-biased estimate of the American option price is deduced

$$
\mathbb{E}^{\star}\left\{Z_{\tau}-M_{\tau} \mid \mathcal{F}_{0}\right\} \leq P_{0}
$$


Note that the hedging martingales used to compute the high-biased estimate and the low-biased estimate can be different. This proposition indicates that whenever one computes a high-biased estimate, it is possible that one can calculate a corresponding low-biased estimate as long as a stopping rule can be realized from, for instance, the least squares method [8]. Though the lower bound estimate is exactly equal to $\mathbb{E}^{\star}\left\{Z_{\tau}\right\}$, we prefer to keep the stopped-martingale term $M_{\tau}$ in order to emphasize its hedging feature and its application to variance reduction. The next result provides two representations for the American option price. They lay the foundation to estimate the price gap and variance of biased estimators in Section 2.2.

Theorem 2 Let $M^{*}$ denote the optimal martingale from the dual formulation (3) and $\tau^{*}$ denotes an optimal stopping time from the primal formulation (1). Then

$$
\begin{array}{ll}
\text { (i) } & P_{0}=\sup _{0 \leq t \leq T}\left(Z_{t}-M_{t}^{*}\right) \text {. } \\
\text { (ii) } & P_{0}=Z_{\tau^{*}}-M_{\tau^{*}}^{*} .
\end{array}
$$

Proof: (i) We first introduce the Snell's envelop process

$$
P_{t}=\operatorname{ess} \sup _{t \leq \tau \leq T} \mathbb{E}^{\star}\left\{Z_{\tau} \mid \mathcal{F}_{t}\right\}
$$

which is a supermartingale of class (D) [9]. Based on the Doob-Meyer decomposition, for any time $t$, $0 \leq t \leq T$, we have

$$
P_{t}=P_{0}+M_{t}^{*}-A_{t}^{*}
$$

where $A_{t}^{*} \geq 0$ is a non-decreasing predictable process vanishing at time zero. Using that $P_{t} \geq Z_{t}$ and the decomposition above, we have

$$
\begin{aligned}
Z_{t}-M_{t}^{*} & \leq P_{t}-M_{t}^{*} \\
& =P_{0}-A_{t}^{*} \\
& \leq P_{0},
\end{aligned}
$$

since $A_{t}^{*} \geq 0$. Taking the supremum over time $0 \leq t \leq T$, we see that $\sup _{0 \leq t \leq T}\left(Z_{t}-M_{t}^{*}\right) \leq P_{0}$. But from Proposition 1 by substituting $M^{*}$ we ensure that almost surely

$$
P_{0}=\sup _{0 \leq t \leq T}\left(Z_{t}-M_{t}^{*}\right)
$$

(ii) On the other hand, the low-biased estimate becomes the American option price when an optimal stopping time $\tau^{*}$ is chosen such that

$$
\mathbb{E}^{\star}\left\{Z_{\tau^{*}}-M_{\tau^{*}}^{*} \mid \mathcal{F}_{0}\right\}=\mathbb{E}^{\star}\left\{Z_{\tau^{*}} \mid \mathcal{F}_{0}\right\}=P_{0}
$$

From Equations (3) and (8), and by the fact that $\sup _{0 \leq t \leq T}\left(Z_{t}-M_{t}^{*}\right) \geq Z_{\tau^{*}}-M_{\tau^{*}}^{*}$, these two random variables have to be equal to the price almost surely

$$
Z_{\tau^{*}}-M_{\tau^{*}}^{*}=\sup _{0 \leq t \leq T}\left(Z_{t}-M_{t}^{*}\right)=P_{0}
$$




\subsection{Price and Variance Errors in American Option Price Estimation}

Based on Proposition 1, for any given martingale $M \in H_{0}^{1}$, one can compute a high-biased and a low-biased estimate for the American option price. We show next that the price error between the high-biased estimate and the American option price, and the variance of high-biased estimator are both highly dependent on the choice of martingales.

Theorem 3 Let $M^{*}$ denotes the optimal martingale as in Theorem 2, and $M \in H_{0}^{1}$ be any given martingale.

(i) The price error between the high-biased estimate $\bar{P}_{0}=\mathbb{E}^{\star}\left\{\sup _{0 \leq t \leq T}\left(Z_{t}-M_{t}\right) \mid \mathcal{F}_{0}\right\}$ and $P_{0}$ is bounded above; namely

$$
\bar{P}_{0}-P_{0} \leq 2 \sqrt{\operatorname{Var}\left(M_{T}^{*}-M_{T}\right)} .
$$

(ii) The variance of $\sup _{0 \leq t \leq T}\left(Z_{t}-M_{t}\right)$ vanishes, if and only if the martingale $M$ is optimal. Proof:

(i) By definition

$$
\begin{aligned}
& \bar{P}_{0}=\mathbb{E}^{\star}\left\{\sup _{0 \leq t \leq T}\left(Z_{t}-M_{t}^{*}+M_{t}^{*}-M_{t}\right) \mid \mathcal{F}_{0}\right\} \\
& \leq P_{0}+\mathbb{E}^{\star}\left\{\sup _{0 \leq t \leq T}\left(M_{t}^{*}-M_{t}\right) \mid \mathcal{F}_{0}\right\} .
\end{aligned}
$$

By $\sup _{0 \leq t \leq T}\left(M_{t}^{*}-M_{t}\right) \leq \sqrt{\left(\sup _{0 \leq t \leq T}\left|M_{t}^{*}-M_{t}\right|\right)^{2}}$ and Jensen's inequality, we deduce

$$
\begin{aligned}
& \mathbb{E}^{\star}\left\{\sup _{0 \leq t \leq T}\left(M_{t}^{*}-M_{t}\right) \mid \mathcal{F}_{0}\right\} \leq \sqrt{\mathbb{E}^{\star}\left\{\left(\sup _{0 \leq t \leq T}\left|M_{t}^{*}-M_{t}\right|\right)^{2} \mid \mathcal{F}_{0}\right\}} \\
& \leq 2 \sqrt{\mathbb{E}^{\star}\left\{\left(M_{T}^{*}-M_{T}\right)^{2} \mid \mathcal{F}_{0}\right\}} .
\end{aligned}
$$

The last inequality is obtained from Doob's maximal inequality [9].

(ii) $(\Rightarrow)$ Since the variance is zero, let $\sup _{0 \leq t \leq T}\left(Z_{t}-M_{t}\right)=C<+\infty$ almost surely for a constant $C$. Then $\mathrm{C}$ is not smaller than the price $P_{0}$ based on the dual formulation. On the other hand, let $\tau^{\varepsilon}$ be

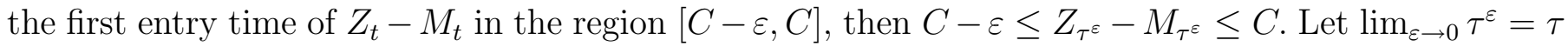
be the limiting stopping time, by dominated convergence Theorem,

$$
C=\mathbb{E}^{\star}\left\{Z_{\tau}-M_{\tau} \mid \mathcal{F}_{0}\right\}=\mathbb{E}^{\star}\left\{Z_{\tau} \mid \mathcal{F}_{0}\right\} .
$$

Hence, from the primal formulation, $\mathrm{C}$ is not larger than the price. Therefore $C$ is equal to the option price $P_{0}$ and by the uniqueness of $M^{*}$ in Theorem 2 (ii) $M$ must be $M^{*}$.

$(\Leftarrow)$ Follows directly from Theorem $2(i)$.

For high-biased estimates, Theorem 3 points out that a martingale closer to the optimal hedging martingale possibly induces a lower upper-bound estimate for the option price and a smaller variance for the high-biased estimator. This property will be illustrated by numerical results implemented in Section 3 and 4.

On the opposite, for the low-biased estimate, the variance of the optimally stopped payoff $Z_{\tau^{*}}$ is $\operatorname{Var}\left\{M_{\tau^{*}}^{*}\right\}$ as seen from Theorem $2(i i)$. We show next that this variance can potentially be reduced by considering an unbiased control variate $Z_{\tau^{*}}-M_{\tau^{*}}$ given a hedging martingale control $M \in H_{0}^{1}$. 
Proposition 4 Given an optimal stopping time $0 \leq \tau^{*} \leq T$ and for any given integrable martingale $M \in H_{0}^{1}$, the variance of the low-biased estimate satisfies

$$
\operatorname{Var}\left\{Z_{\tau^{*}}-M_{\tau^{*}}\right\} \leq \operatorname{Var}\left\{M_{T}^{*}-M_{T}\right\}
$$

Proof:

$$
\begin{aligned}
& \operatorname{Var}\left\{Z_{\tau^{*}}-M_{\tau^{*}}\right\}=\mathbb{E}^{\star}\left\{\left(Z_{\tau^{*}}-M_{\tau^{*}}-P_{0}\right)^{2} \mid \mathcal{F}_{0}\right\} \\
& =\mathbb{E}^{\star}\left\{\left(M_{\tau^{*}}^{*}-M_{\tau^{*}}\right)^{2} \mid \mathcal{F}_{0}\right\} \\
& \leq \operatorname{Var}\left\{M_{T}^{*}-M_{T}\right\} .
\end{aligned}
$$

When an arbitrary stopping time is used, the error bound and the variance between its low-biased estimate and the American option price are not given explicitly here. However, an asymptotic result on the least squares method [8] shows that the optimal stopping rule or the free boundary can be realized when the number of simulated trajectory and the number of basis used to estimate the continuation value go to infinity.

To summarize we observe an asymmetric effect for pricing American options from the point of view of variance reduction. A better hedging martingale provides a smaller variance for high and low biased estimators. But it preserves the bias for the low-biased estimate while shrinking the high bias price gap as the hedging martingale approaches to the optimal martingale.

\section{Numerical Results I: One-Dimensional Case}

This section concerns a typical American put option pricing problem under the Black-Scholes model. That is, under the risk-neutral probability measure the underlying risky stock price $S_{t}$ is governed by the geometric Brownian motion

$$
d S_{t}=r S_{t} d t+\sigma S_{t} d W_{t}
$$

where $r$ is the risk-free interest rate and $W_{t}$ is a Brownian motion.

The American put option price at time $t$ is given as an optimal stopping problem

$$
P\left(t, S_{t}\right)=\operatorname{ess} \sup _{t \leq \tau \leq T} \mathbb{E}^{\star}\left\{e^{-r(\tau-t)}\left(K-S_{\tau}\right)^{+} \mid S_{t}\right\}
$$

with $\tau$ being a bounded stopping time between the current time $t$ and the maturity $T$, and where we have used the Markov property of $S_{t}$.

Proposition 5 The optimal stopping time $0 \leq \tau^{*} \leq T$ of the American option price $P\left(0, S_{0}\right)$ is the first time which maximizes the hedging error. Namely for any time $0 \leq u<\tau^{*}$

$$
e^{-r u}\left(K-S_{u}\right)^{+}-M_{u}^{*}<\sup _{0 \leq t \leq T}\left(e^{-r t}\left(K-S_{t}\right)^{+}-M_{t}^{*}\right),
$$

but the equality holds when $t=\tau^{*}$. Namely

$$
\tau^{*}=\inf \left\{0 \leq t \leq T, e^{-r t}\left(K-S_{t}\right)^{+}-M_{t}^{*}=P\left(0, S_{0}\right)\right\} .
$$

Proof: For any time $0 \leq t<\tau^{*}$, the exercise payoff must be less than the American option price $\left(K-S_{t}\right)^{+}<P\left(t, S_{t}\right)$ and

$$
e^{-r t}\left(K-S_{t}\right)^{+}-M_{t}^{*}<e^{-r t} P\left(t, S_{t}\right)-M_{t}^{*}=P\left(0, S_{0}\right)-A_{t}^{*} \leq P\left(0, S_{0}\right)
$$


by the Doob-Meyer decomposition (6). We see that the discounted payoff $e^{-r t}\left(K-S_{t}\right)^{+}$is superhedged by the hedging portfolio $P\left(0, S_{0}\right)+M_{t}^{*}$ at any time prior to the optimal stopping time. Combining with Theorem 2 (ii) we conclude that $\tau^{*}$ is the first time maximizing the hedging error $Z_{t}-M_{t}^{*}$. If $\tau^{*}=0$, it is a trivial case.

\subsection{Hedging Martingales}

It is known that there is no closed-form solution for the American option price $P(t, x)$ given by $(10)$. Rogers [10] introduced the counterpart European put option price, denoted by $P_{E}$, and constructed the hedging martingale $e^{-r t} P_{E}\left(t, S_{t}\right)-P_{E}\left(0, S_{0}\right)$. This choice is useful because $P_{E}$ admits a closed-form solution, known as the Black-Scholes formula for put options. Instead, we write an equivalent integral representation of that hedging martingale as

$$
M\left(P_{E} ; t\right)=\int_{0}^{t} e^{-r s} \frac{\partial P_{E}}{\partial x}\left(s, S_{s}\right) \sigma S_{s} d W_{s},
$$

obtained by an application of Ito's Lemma to $e^{-r t} P_{E}\left(t, S_{t}\right)$. The main advantage of (12) is that any

approximate American option price $\tilde{P}$ can constitute an integral martingale $M(\tilde{P} ; t)$ in addition to $P_{E}$, without requiring that $e^{-r t} \tilde{P}_{t}$ be a martingale. Algorithms to computer the high and low biased estimates for the American put option are based on Proposition 1. The Monte Carlo estimator for the high-biased estimator is

$$
\frac{1}{N} \sum_{i=1}^{N} \sup _{0 \leq t \leq T}\left(e^{-r t}\left(K-S_{t}^{(i)}\right)^{+}-M^{(i)}(\tilde{P} ; t)\right),
$$

and for the low-biased estimator is

$$
\frac{1}{N} \sum_{i=1}^{N}\left[e^{-r \tau}\left(K-S_{\tau}^{(i)}\right)^{+}-M^{(i)}(\tilde{P} ; \tau)\right],
$$

where the approximation $\tilde{P}$ shall be easy to compute, for example the counterpart European option price $P_{E}$ or the quadratic approximation $P_{B A W}$, introduced by Barone-Adesi and Whaley [1]. The total number of i.i.d. trajectories is denoted by $N$, the superscript $(i)$ denoting the $i$-th replication, and $\tau$ denoting a stopping rule, obtained by least squares method [8].

Based on the solution of an elliptic-type variational inequalities shown in equation (27) in the Appendix, the approximation $P_{B A W}$ admits the following analytic solution

$$
P_{B A W}(t, x)= \begin{cases}\lambda x^{\alpha}+P_{E}(t, x), & x>x^{*}(t) \\ K-x, & x \leq x^{*}(t)\end{cases}
$$

where $P_{E}(t, x)$ denotes the counterpart European put option price, and where the approximate free boundary $x^{*}(t)$ solves a nonlinear algebraic equation

$$
x^{*}(t)=|\alpha| \frac{K-P_{E}\left(t, x^{*}\right)}{\frac{\partial P_{E}\left(t, x^{*}(t)\right)}{\partial x}+1+|\alpha|},
$$

with parameters

$$
\begin{aligned}
& \alpha=\frac{1-\frac{2 r}{\sigma^{2}}-\sqrt{\left(1-\frac{2 r}{\sigma^{2}}\right)^{2}+\frac{8(\kappa r+1)}{\kappa \sigma^{2}}}}{2}, \\
& \lambda=\frac{K-x^{*}(t)-P_{E}\left(t, x^{*}(t)\right)}{\left(x^{*}(t)\right)^{\alpha}} .
\end{aligned}
$$


It is shown in Lemma 7 in the Appendix that the discounted approximate price $e^{-r t} P_{B A W}$ is not a martingale nor a supermartingale. It cannot be used in Proposition 1 to estimate American option prices. However the stochastic integral $M\left(P_{B A W} ; t\right)$ is guaranteed to be a martingale. The martingale property of stochastic integrals not only provides a larger class for the computational purpose, but also it is a clear demonstration of delta hedging strategy used in dynamic trading.

We are now ready to compare these hedging martingales $M\left(P_{E} ; t\right)$ and $M\left(P_{B A W} ; t\right)$ when estimating high and low biased solutions for American option prices. In Table 1, parameters of one-dimensional American put options are as follows: the strike price $K=100$, the risk free interest rate $r=6 \%$, maturity $T=0.5$ year, and the volatility $\sigma=0.4$. The initial stock price $S_{0}$ is varying from 80 to 120 . We run $N=5000$ sample paths, and for each trajectory we use the discretized time step $\Delta t=0.001$. The true prices shown on the column 5 in Table 1 are identical to the example in [10].

Low biased estimates and their standard errors for American option prices are illustrated between the second column and the fourth column. Results in column 2 are calculated from the least squares algorithm [8] where there is no hedging martingales within the price estimator. Column 3 and column 4 illustrate effects of martingale controls $M\left(P_{E} ; t\right)$ and $M\left(P_{B A W} ; t\right)$ respectively, under the least squares method. We make the following observations.

- First, these control variates are unbiased to least squares estimators but the standard errors with martingales are both greatly reduced compared with the least squares estimators. The variance reduction ratios are roughly between 60 and 200. As the low-biased estimates should behave, sample means within column 2 to column 4 are all smaller than the true prices shown in the fifth column.

- Second, the algorithm using $M\left(P_{B A W} ; t\right)$ improves the precision of the low biased estimates obtained from $M\left(P_{E} ; t\right)$ as the variance produced by $M\left(P_{B A W} ; t\right)$ is smaller than $M\left(P_{E} ; t\right)$ except when $S_{0}=95$. Column 6 and 7 illustrate high-biased estimates based on the algorithm using the martingales $M\left(P_{B A W} ; t\right)$ and $M\left(P_{E} ; t\right)$ respectively. Compared to the true price in column 5 , sample means obtained from these martingales are all high biased and the price gap in column 6 is smaller than those in column 7. Moreover the standard errors in column 6 are all smaller than those in column 7. This justifies the asymmetric property between the bias and variance in Theorem 3 . Namely a small variance implies a small bias.

We do not report MAD (mean absolute deviation from the mean) defined in [10] as we now have both high and low biased price estimates for which the actual option price is in between. By cross comparison between columns 3-7 and columns 4-6, we find that $P_{B A W}$ does provides a better approximation than $P_{E}$ as $P_{B A W}$ produces smaller variances than $P_{E}$ does.

\subsection{Errors in Delta Approximations}

As suggested in Theorem 3, a martingale close to the optimal one will induce a smaller price gap, and a smaller variance for the high biased estimate. We measure the distance between two martingales by using the second moment or the variance. It is shown in [2] that the variance is bounded above:

$$
\mathbb{E}^{\star}\left\{\left(M_{T}^{*}-M(\tilde{P} ; T)\right)^{2} \mid \mathcal{F}_{0}\right\} \leq C \sqrt{\int_{0}^{T} \mathbb{E}^{\star}\left\{\left(\frac{\partial P}{\partial x}-\frac{\partial \tilde{P}}{\partial x}\right)^{2}\left(t, S_{t}\right) \mid \mathcal{F}_{0}\right\} d t}
$$

where the constant $C$ depends only on the initial stock price $S_{0}$ and the volatility $\sigma$. The mean square of the delta difference $\frac{\partial P}{\partial x}-\frac{\partial \tilde{P}}{\partial x}$ is crucial to control the distance between hedging martingales. There 
Table 1: Numerical Results I. Comparisons of high-biased price estimates (Columns 6-8), lowbiased price estimates (Columns 2-4), and actual American option prices (Column 5). Two hedging martingales $M_{t}\left(P_{E}\right)$ and $M_{t}\left(P_{B A W}\right)$ are constructed from the counterpart European option price $P_{E}$ and the quadratic approximation $P_{B A W}$ respectively. Model parameters are chosen as in [10]; $K=100, r=0.06, T=0.5, \sigma=0.4$, with various initial stock prices ranging from 80 to 120 . Monte Carlo simulations are implemented under the sample size $N=5000$ and 500 discrete time steps corresponding to $\Delta t=.001$.

\begin{tabular}{|c|c|c|c|c|c|c|c|}
\hline$S_{0}$ & LSM & $M_{t}\left(P_{E}\right)$ & $M_{t}\left(P_{B A W}\right)$ & True Price & $M_{t}\left(P_{B A W}\right)$ & $M_{t}\left(P_{E}\right)$ & $S M_{t}$ \\
\hline 80 & 21.522 & 21.513 & 21.592 & 21.606 & 21.754 & 21.947 & 22.637 \\
& $(0.1507)$ & $(0.0131)$ & $(0.0108)$ & & $(0.0097)$ & $(0.0107)$ & $(0.0092)$ \\
\hline 85 & 17.907 & 17.952 & 17.999 & 18.037 & 18.203 & 18.325 & 18.793 \\
& $(0.1631)$ & $(0.0138)$ & $(0.0125)$ & & $(0.0121)$ & $(0.0128)$ & $(0.0093)$ \\
\hline 90 & 14.817 & 14.874 & 14.845 & 14.919 & 15.073 & 15.132 & 15.482 \\
& $(0.1706)$ & $(0.0155)$ & $(0.0139)$ & & $(0.0129)$ & $(0.0143)$ & $(0.0085)$ \\
\hline 95 & 12.141 & 12.163 & 12.202 & 12.231 & 12.371 & 12.391 & 12.649 \\
& $(0.1640)$ & $(0.0153)$ & $(0.0155)$ & & $(0.0138)$ & $(0.0148)$ & $(0.0075)$ \\
\hline 100 & 9.993 & 9.868 & 9.880 & 9.946 & 10.090 & 10.147 & 10.270 \\
& $(0.1585)$ & $(0.0158)$ & $(0.0150)$ & & $(0.0144)$ & $(0.0153)$ & $(0.0066)$ \\
\hline 105 & 8.214 & 8.023 & 8.026 & 8.028 & 8.140 & 8.181 & 8.275 \\
& $(0.1497)$ & $(0.0166)$ & $(0.0154)$ & & $(0.0146)$ & $(0.0151)$ & $(0.0056)$ \\
\hline 110 & 6.205 & 6.355 & 6.433 & 6.435 & 6.564 & 6.612 & 6.625 \\
& $(0.1304)$ & $(0.0160)$ & $(0.0153)$ & & $(0.0143)$ & $(0.0149)$ & $(0.0048)$ \\
\hline 115 & 5.126 & 5.085 & 5.055 & 5.127 & 5.256 & 5.269 & 5.280 \\
& $(0.1219)$ & $(0.0157)$ & $(0.0150)$ & & $(0.0135)$ & $(0.0141)$ & $(0.0041)$ \\
\hline 120 & 4.230 & 4.029 & 4.039 & 4.061 & 4.184 & 4.198 & 4.180 \\
& $(0.1162)$ & $(0.0147)$ & $(0.0143)$ & & $(0.0128)$ & $(0.0134)$ & $(0.0033)$ \\
\hline
\end{tabular}

is no guarantee that a better price approximation provides a better delta approximation. The study of delta approximation for European option prices can be found in [2] under multiscale stochastic volatility models. It remains a challenging task to study delta approximation for American options. At least from numerical results one can see a strong empirical support that on average the approximate price $P_{B A W}$ provides a better delta approximation than the European option price $P_{E}$. Note that these comparisons are useful to justify the effectiveness of price approximations.

\subsection{Hedging Supermartingales}

We should mention an important result from Haugh and Kogan [7]. Rather than martingales, they use supermartingales to obtain high-biased estimates for the American option price

$$
P\left(0, S_{0}\right)=\inf _{\bar{M} \in \bar{H}^{1}} \mathbb{E}^{\star}\left\{\sup _{0 \leq t \leq T}\left(e^{-r t}\left(K-S_{t}\right)^{+}-\bar{M}_{t}\right) \mid \mathcal{F}_{0}\right\}+\bar{M}_{0}
$$


where $\bar{H}^{1}=\left\{\left(\bar{M}_{t}\right)_{0 \leq t \leq T}\right.$ : supermartingale with $\left.\sup _{0 \leq t \leq T}\left|\bar{M}_{t}\right| \in L^{1}\right\}$. It is shown in [7] that the infimum can be obtained by choosing $\bar{M}_{t}^{*}=e^{-r t} P\left(t, S_{t}\right)$ such that

$$
P\left(0, S_{0}\right)=\mathbb{E}^{\star}\left\{\sup _{0 \leq t \leq T}\left(e^{-r t}\left(K-S_{t}\right)^{+}-\left(\bar{M}_{t}^{*}-\bar{M}_{0}^{*}\right)\right) \mid \mathcal{F}_{0}\right\}
$$

Proposition 6 For the American option pricing problem (10), the supermartingale characterization (16) by Haugh and Kogan [7], and the martingale characterization (2) by Rogers [10] are the same in the following sense: at the optimal stopping time $\tau^{*}$, defined in Proposition 5, the optimizer of supermartingale $\bar{M}_{\tau^{*}}^{*}-\bar{M}_{0}^{*}$ is equal to the optimizer of martingale $M_{\tau^{*}}^{*}$.

Proof:

1. We first show that the hedging supermartingale representation holds almost surely:

$$
P\left(0, S_{0}\right)=\sup _{0 \leq t \leq T}\left(e^{-r t}\left(K-S_{t}\right)^{+}-\left(e^{-r t} P\left(t, S_{t}\right)-P\left(0, S_{0}\right)\right)\right) .
$$

By Doob-Meyer decomposition as in (6), we obtain

$$
e^{-r t} P\left(t, S_{t}\right)-P\left(0, S_{0}\right)=M_{t}^{*}-A_{t}^{*}
$$

such that

$$
\begin{aligned}
& \sup _{0 \leq t \leq T}\left(e^{-r t}\left(K-S_{t}\right)^{+}-\left(e^{-r t} P\left(t, S_{t}\right)-P\left(0, S_{0}\right)\right)\right) \\
& \geq \sup _{0 \leq t \leq T}\left(e^{-r t}\left(K-S_{t}\right)^{+}-M_{t}^{*}\right)=P\left(0, S_{0}\right)
\end{aligned}
$$

by $A_{t}^{*} \geq 0$ and Theorem 2 (ii). The supremum of hedging errors by supermartingales $e^{-r t} P\left(t, S_{t}\right)$ is no less than the true price $P\left(0, S_{0}\right)$. This contradicts Equation (17) unless the supremum is the price almost surely. Thus we obtain Equation (18).

2. Substituting the decomposition (19) in (18), we deduce

$$
P\left(0, S_{0}\right)=\sup _{0 \leq t \leq T}\left(e^{-r t}\left(K-S_{t}\right)^{+}-\left(M_{t}^{*}-A_{t}^{*}\right)\right)
$$

By Proposition 5 the optimal stopping time $\tau^{*}$ is the first time such that

$$
P\left(0, S_{0}\right)=e^{-r \tau^{*}}\left(K-S_{\tau^{*}}\right)^{+}-M_{\tau^{*}}^{*},
$$

with $A_{\tau^{*}}$. Hence $\bar{M}_{\tau^{*}}^{*}-\bar{M}_{0}^{*}=e^{-r t} P\left(t, S_{t}\right)-P\left(0, S_{0}\right)=M_{\tau^{*}}^{*}$.

Given a supermartingale $\bar{M}_{t} \in \bar{H}^{1}$, one can calculate a high-biased estimate for the American option price

$$
P\left(0, S_{0}\right) \leq \mathbb{E}^{\star}\left\{\sup _{0 \leq t \leq T}\left(e^{-r t} H\left(S_{t}\right)-\left(\bar{M}_{t}-\bar{M}_{0}\right)\right)\right\} .
$$

As revealed from Lemma 7, the approximate early exercise premium with a weighted discount factor is a supermartingale. So we propose the supermartingale $S M_{t}=e^{-r t} P_{E}\left(t, S_{t}\right)+e^{-\left(r+\frac{1}{\kappa(t)}\right) t} V\left(S_{t}\right)$, 
where the early exercise premium approximation is given by $V\left(S_{t}\right)=P_{B A W}\left(t, S_{t}\right)-P_{E}\left(t, S_{t}\right)$, and we construct the following supermartingale control

$$
S M_{t}-S M_{0}=e^{-r t} P_{E}\left(t, S_{t}\right)+e^{-\left(r+\frac{1}{\kappa(t)}\right) t} V\left(S_{t}\right)-P_{B A W}\left(0, S_{0}\right) .
$$

Based on the high-biased estimate

$$
\mathbb{E}^{\star}\left\{\sup _{0 \leq t \leq T}\left(e^{-r t} H\left(S_{t}\right)-\left(S M_{t}-S M_{0}\right)\right)\right\},
$$

numerical results are shown in the last column of Table 1. Because the supermartingale property of $e^{-\left(r+\frac{1}{\kappa(t)}\right) t} V\left(S_{t}\right)$, the bias estimated is larger than those obtained from martingale control $M\left(P_{E} ; t\right)$. But it is somehow surprising to see that the standard errors obtained from the supermartingale $\mathrm{SM}_{t}$ algorithm are the smallest compared to those obtained from $M\left(P_{B A W} ; t\right)$ and $M\left(P_{E} ; t\right)$. Though this phenomenon is not in contradiction with Theorem 3 for martingales, it remains to investigate further how to construct suitable supermartingale estimators in order to reduce the price gap while keeping small variance.

\section{Numerical Results II: Stochastic Volatility}

\subsection{Multiscale Stochastic Volatility Models}

Following [5], we consider the following class of multiscale stochastic volatility models, under a riskneutral pricing probability measure $\mathbb{P}^{\star}$ parametrized by the combined market prices of volatility risk $\left(\Lambda_{1}, \Lambda_{2}\right)$ :

$$
\begin{aligned}
d S_{t} & =r S_{t} d t+\sigma_{t} S_{t} d W_{t}^{(0) \star} \\
\sigma_{t} & =f\left(Y_{t}, Z_{t}\right) \\
d Y_{t} & =\left[\frac{1}{\varepsilon} c_{1}\left(Y_{t}\right)+\frac{g_{1}\left(Y_{t}\right)}{\sqrt{\varepsilon}} \Lambda_{1}\left(Y_{t}, Z_{t}\right)\right] d t+\frac{g_{1}\left(Y_{t}\right)}{\sqrt{\varepsilon}}\left(\rho_{1} d W_{t}^{(0) \star}+\sqrt{1-\rho_{1}^{2}} d W_{t}^{(1) \star}\right) \\
d Z_{t} & =\left[\delta c_{2}\left(Z_{t}\right)+\sqrt{\delta} g_{2}\left(Z_{t}\right) \Lambda_{2}\left(Y_{t}, Z_{t}\right)\right] d t \\
& +\sqrt{\delta} g_{2}\left(Z_{t}\right)\left(\rho_{2} d W_{t}^{(0) \star}+\rho_{12} d W_{t}^{(1) \star}+\sqrt{1-\rho_{2}^{2}-\rho_{12}^{2}} d W_{t}^{(2) \star}\right)
\end{aligned}
$$

where $S_{t}$ is the underlying asset price process with a constant risk-free interest rate $r$. The random stochastic volatility $\sigma_{t}$ is driven by two stochastic processes $Y_{t}$ and $Z_{t}$ varying on the time scales $\varepsilon$ and $1 / \delta$, respectively ( $\varepsilon$ is intended to be a short time scale while $1 / \delta$ is thought as a longer time scale). The vector $\left(W_{t}^{(0) \star}, W_{t}^{(1) \star}, W_{t}^{(2) \star}\right)$ consists of three independent standard Brownian motions. The instant correlation coefficients $\rho_{1}, \rho_{2}$, and $\rho_{12}$ satisfy $\left|\rho_{1}\right|<1$ and $\left|\rho_{2}^{2}+\rho_{12}^{2}\right|<1$. The volatility function $f$ is assumed to be bounded and bounded away from zero to avoid degeneracy, though these assumptions are not crucial and can be relaxed to accommodate for instance Heston-type models with a CIR stochastic volatility factor. The coefficient functions of $Y_{t}$, namely $c_{1}$ and $g_{1}$, are assumed to be such that under the physical probability measure $\left(\Lambda_{1}=\Lambda_{2}=0\right), Y_{t}$ is ergodic. The Ornstein-

Uhlenbeck (OU) process is a typical example by defining $c_{1}(y)=m_{1}-y$ and $g_{1}(y)=\nu_{1} \sqrt{2}$ such that $1 / \varepsilon$ is the rate of mean reversion, $m_{1}$ is the long run mean, and $\nu_{1}$ is the long run standard deviation. Its invariant distribution is $\mathcal{N}\left(m_{1}, \nu_{1}^{2}\right)$. 
The coefficient functions of $Z_{t}$, namely $c_{2}$ and $g_{2}$ are assumed to be smooth enough in order to satisfy existence and uniqueness conditions for diffusions. The combined risk premia $\Lambda_{1}$ and $\Lambda_{2}$ are assumed to be smooth, bounded, and depending on the variables $y$ and $z$ only. Within this setup, the joint process $\left(S_{t}, Y_{t}, Z_{t}\right)$ is Markovian. We refer to [5] for a detailed discussion on this class of models.

Under the stochastic volatility models considered, the American option price at time 0 with an integrable payoff function $H$ is given by:

$$
P^{\varepsilon, \delta}(t, x, y, z)=\mathrm{ess} \sup _{t \leq \tau \leq T} \mathbb{E}^{\star}\left\{e^{-r(\tau-t)} H\left(S_{\tau}\right) \mid S_{t}=x, Y_{t}=y, Z_{t}=z\right\}
$$

where $\tau$ denotes any stopping time greater than or equal to $t$, bounded by $T$, and is adapted to the completion of the natural filtration generated by Brownian motions $\left(W_{t}^{(0) \star}, W_{t}^{(1) \star}, W_{t}^{(2) \star}\right)$. We consider a typical American put option pricing problem, namely $H(x)=(K-x)^{+}$.

\subsection{Projected Hedging Martingales from Asymptotic Expansion}

As shown in Proposition 1 one needs to construct a martingale in order to calculate the high and low biased estimates for the American option price. Under the Black-Scholes model, the volatility is assumed to be constant. We have observed in previous section that the use of counterpart discounted European option price, which admits a closed-form solution, as a martingale is adequate. Under stochastic volatility models there no longer exists a closed-form solution for the European option price. A martingale being a discounted European option price must be computed by for example another Monte Carlo simulation. This computation of Monte Carlo on Monte Carlo is typically very time consuming. To overcome this difficulty, the authors, in [2], proposed the following: first apply Ito's lemma to $e^{-r t} P\left(t, S_{t}, Y_{t}, Z_{t}\right)$ and integrate from time 0 to $\tau^{\star}$. Then a hedging martingale consists of three parts $M_{t}(\tilde{P})=M_{0}(\tilde{P} ; t)+M_{1}(\tilde{P} ; t)+M_{2}(\tilde{P} ; t)$, where $\tilde{P}\left(s, S_{s}, Y_{s}, Z_{s}\right)$ denotes any approximation to the true model price $P^{\varepsilon, \delta}\left(s, S_{s}, Y_{s}, Z_{s}\right)$ given by $(21)$, the three martingales being given by

$$
\begin{aligned}
& M_{0}(\tilde{P} ; t)=\int_{0}^{t} e^{-r s} \frac{\partial \tilde{P}}{\partial x}\left(s, S_{s}, Y_{s}, Z_{s}\right) f\left(Y_{s}, Z_{s}\right) S_{s} d W_{s}^{(0) *} \\
& M_{1}(\tilde{P} ; t)=\frac{1}{\sqrt{\varepsilon}} \int_{0}^{t} e^{-r s} \frac{\partial \tilde{P}}{\partial y}\left(s, S_{s}, Y_{s}, Z_{s}\right) g_{1}\left(Y_{s}\right) d \tilde{W}_{s}^{(1) *} \\
& M_{2}(\tilde{P} ; t)=\sqrt{\delta} \int_{0}^{t} e^{-r s} \frac{\partial \tilde{P}}{\partial z}\left(s, S_{s}, Y_{s}, Z_{s}\right) g_{2}\left(Z_{s}\right) d \tilde{W}_{s}^{(2) *}
\end{aligned}
$$

where the Brownian motions are defined by

$$
\begin{aligned}
& \tilde{W}_{s}^{(1) \star}=\rho_{1} W_{s}^{(0) \star}+\sqrt{1-\rho_{1}^{2}} W_{s}^{(1) \star} \\
& \tilde{W}_{s}^{(2) \star}=\rho_{2} W_{s}^{(0) \star}+\rho_{12} W_{s}^{(1) \star}+\sqrt{1-\rho_{1}^{2}-\rho_{12}^{2}} W_{s}^{(2) \star} .
\end{aligned}
$$

In general, the hedging martingale can include control parameters $\lambda_{0}, \lambda_{1}, \lambda_{2}$ such that

$$
M_{t}\left(\tilde{P} ; \lambda_{0}, \lambda_{1}, \lambda_{2}\right)=\lambda_{0} M_{0}(\tilde{P} ; t)+\lambda_{1} M_{1}(\tilde{P} ; t)+\lambda_{2} M_{2}(\tilde{P} ; t) .
$$

A projected martingale considered here is constructed from a combination of hedging martingales and asymptotic methods.

We now focus on an approximation of the American option price under stochastic volatility models. 
Table 2: Parameters used in the two-factor stochastic volatility model (20).

\begin{tabular}{|c|c|c|c|c|c|c|c|c|c|c|}
\hline$r$ & $m_{1}$ & $m_{2}$ & $\nu_{1}$ & $\nu_{2}$ & $\rho_{1}$ & $\rho_{2}$ & $\rho_{12}$ & $\Lambda_{1}$ & $\Lambda_{2}$ & $f(y, z)$ \\
\hline $10 \%$ & -1 & -1 & 1 & 1 & -0.3 & -0.3 & 0 & 0 & 0 & $\exp (y+z)$ \\
\hline
\end{tabular}

Table 3: Initial conditions and American put option parameters.

\begin{tabular}{|c|c|c|c|c|}
\hline$\$ S_{0}$ & $Y_{0}$ & $Z_{0}$ & $\$ K$ & $T$ years \\
\hline 90 & -1 & -1 & 100 & 1 \\
\hline
\end{tabular}

When the time scales $1 / \varepsilon$ and $\delta$ are well separated; namely $0<\varepsilon, \delta \ll 1$, the American option price $P^{\varepsilon, \delta}\left(t, S_{t}, Y_{t}, Z_{t}\right)$ admits an asymptotic expansion following the arguments in [4,5]. The leading order term in the expansion is given by

$$
P_{0}\left(t, S_{t} ; \bar{\sigma}\left(Z_{t}\right)\right)=\operatorname{ess} \sup _{t \leq \tau \leq T} E\left\{e^{-r(\tau-t)} H\left(\bar{S}_{\tau}\right) \mid \bar{S}_{t}=S_{t}\right\}
$$

where the homogenized stock price $\bar{S}_{t}$ follows a geometric Brownian motion with the averaged volatility $\bar{\sigma}(z)=\sqrt{\left\langle f^{2}(y, z)\right\rangle_{Y}}$ and $\langle\cdot\rangle_{Y}$ denotes the averaging with respect to the invariant distribution of the fast varying process $Y$. Note that because the homogenized American option price $P_{0}\left(t, S_{t} ; \bar{\sigma}\left(Z_{t}\right)\right)$ does not depend on the $Y$ process, $M_{1}\left(P_{0} ; t\right)$ shown in $(23)$ is omitted (in fact it can be shown that the next term of order $\sqrt{\varepsilon}$ in the expansion is also independent of $y$ so that $M_{1}\left(P_{0} ; t\right)$ would only contribute at the order $\sqrt{\varepsilon}$ which justifies this omission). Since $M_{2}\left(P_{0} ; t\right)$ in $(24)$ is of small order $\sqrt{\delta}$, this martingale is also neglected. As a result, the hedging martingale $(25)$ is reduced to $M_{t}\left(P_{0}\right)=\lambda_{0} M_{0}\left(P_{0} ; t\right)$.

As an American option under constant volatility, the homogenized American option $P_{0}\left(t, x ; \bar{\sigma}\left(Z_{t}\right)\right)$ does not admit a closed-form solution. We follow the discuss in Section 3 and use approximations to $P_{0}(t, x)$ in order to construct hedging martingales as stochastic integrals such as $M_{t}=M_{0}\left(P_{E} ; t\right)$ or $M_{t}=M_{0}\left(P_{B A W} ; t\right)$, in which we do not pursue the optimal $\lambda_{0}$ but simply take $\lambda_{0}=1$ as it is found near one in [10] under the Black- Scholes model. As a result, we can use the same algorithm in $(13,14)$ to estimate American option prices under stochastic volatility models, though a stopping rule must be calculated by for example the least squares method.

We consider American put options under two factor stochastic volatility models, specified in Table 2 and Table 3. Results of high and low biased estimates to price American put options are illustrated in Table 4 with various time scale parameters $\varepsilon$ and $\delta$. The discrete time step size is $\Delta t=.001$ and the total sample size is $N=5000$.

Similar observations from numerical results in Table 4 can be made as those from Table 1 . Lowbiased estimates are all unbiased to the estimator using the least squares method. We see that the control $M_{t}\left(P_{B A W}\right)$ provides slightly better variance reduction ratios than $M_{t}\left(P_{E}\right)$ does. High-biased estimates obtained from $M_{t}\left(P_{B A W}\right)$ outperform those from $M_{t}\left(P_{E}\right)$ because they provide both smaller biases and errors.

\section{Conclusion}

We have shown that hedging martingales are crucial for both primal and dual approaches to estimating American option prices by Monte Carlo simulations. The hedging martingales can be constructed from any price approximation to American option prices. We uncovered the following asymmetric relation 
Table 4: Numerical Results: Comparison of Low-Biased estimates and High-Biased estimates with some projected hedging martingales under different sets of time scales.

\begin{tabular}{|c|c|c|c|c|c|c|}
\hline $1 / \varepsilon$ & $\delta$ & $L S M($ primal $)$ & $M_{t}\left(P_{E}\right)($ primal $)$ & $M_{t}\left(P_{B A W}\right)($ primal $)$ & $M_{t}\left(P_{B A W}\right)($ dual $)$ & $M_{t}\left(P_{E}\right)($ dual $)$ \\
\hline 100 & 0.01 & 21.83 & 21.70 & 21.69 & 22.29 & 22.89 \\
& & $(0.241)$ & $(0.034)$ & $(0.025)$ & $(0.024)$ & $(0.037)$ \\
\hline 75 & \multirow{2}{*}{0.1} & 21.69 & 21.57 & 21.57 & 22.33 & 22.86 \\
& & $(0.238)$ & $(0.034)$ & $(0.027)$ & $(0.027)$ & $(0.039)$ \\
\hline 50 & 1 & 21.90 & 21.53 & 21.51 & 22.37 & 22.91 \\
& & $(0.242)$ & $(0.040)$ & $(0.033)$ & $(0.033)$ & $(0.042)$ \\
\hline 25 & \multirow{2}{*}{10} & 21.10 & 21.38 & 21.31 & 22.29 & 22.94 \\
& & $(0.267)$ & $(0.055)$ & $(0.048)$ & $(0.043)$ & $(0.051)$ \\
\hline
\end{tabular}

between the biases and variances for primal approach and dual approach: the dual approach ensures that a good hedging martingale induces a lower high-biased estimate with a smaller variance, while the primal approach ensures that a good hedging martingale reduces the variance for a low-biased estimate given a stopping time. Moreover under more realistic multifactor stochastic volatility models, we propose a projected hedging martingale obtained by an asymptotic expansion. Numerical results demonstrate the robustness and effeciency of this method.

\section{A Appendix}

The approximate American option price $P_{B A W}(t, x)$ is equal to the sum of the counterpart European option price denoted by $P_{E}(t, x)$ and an approximate early exercise premium $V(x ; t)$, where $V(x ; t)$ solves an elliptic-type variational inequalities

$$
\left\{\begin{array}{l}
\mathcal{A}_{B S}(\sigma) V(x ; t)-\left(r+\frac{1}{\kappa(t)}\right) V(x ; t) \leq 0 \\
V(x ; t) \geq(K-x)^{+}-P_{E}(t, x) \\
\left(\mathcal{A}_{B S}(\sigma) V(x ; t)-\left(r+\frac{1}{\kappa(t)}\right) V(x ; t)\right) \cdot\left(V(x ; t)-(K-x)^{+}+P_{E}(t, x)\right)=0
\end{array}\right.
$$

with the differential operator $\mathcal{A}_{B S}(\sigma)=\frac{\sigma^{2} x^{2}}{2} \frac{\partial^{2}}{\partial x^{2}}+r x \frac{\partial}{\partial x}-r \cdot$

\section{Lemma 7}

(i) $e^{-r t} V\left(S_{t}\right)$ is not a supermartingale, nor $e^{-r t} P_{B A W}\left(t, S_{t}\right)$.

(ii) $e^{-\left(r+\frac{1}{\kappa(t)}\right) t} V\left(S_{t}\right)$ is a supermartingale, where $\kappa(t)=\frac{e^{r(T-t)}-1}{r} \geq 0$ for each $t \in[0, T]$.

Proof:

1. For any time $t<\tau^{\star}$,

$$
\begin{aligned}
d\left[e^{-r t} V\left(S_{t}\right)\right] & =e^{-r t}\left[\mathcal{A}_{B S}(\sigma) V\left(S_{t}\right)-r V\left(S_{t}\right)\right] d t+e^{-r t} \frac{\partial V}{\partial x}\left(S_{t}\right) \sigma S_{t} d W_{t} \\
& =e^{-r t} \frac{V\left(S_{t}\right)}{\kappa(t)} d t+e^{-r t} \frac{\partial V}{\partial x}\left(S_{t}\right) \sigma S_{t} d W_{t}
\end{aligned}
$$

Since the drift term is greater than zero, $e^{-r t} V\left(S_{t}\right)$ is not a supermartingale. Because $e^{-r t} P_{B A W}\left(t, S_{t}\right)=$ $e^{-r t} P_{E}\left(t, S_{t}\right)+e^{-r t} V\left(S_{t}\right)$, it can not be a supermartingale as well. 
2. By an application of Ito's lemma to $e^{-\left(r+\frac{1}{\kappa(s)}\right) s} V\left(S_{s}\right)$, we obtain

$$
\begin{aligned}
& d\left[e^{-\left(r+\frac{1}{\kappa(s)}\right) s} V\left(S_{s}\right)\right] \\
= & e^{-\left(r+\frac{1}{\kappa(s)}\right) s}\left\{\left(\frac{\sigma^{2} S_{s}^{2}}{2} \frac{\partial^{2} V}{\partial x^{2}}\left(S_{s}\right)+r S_{s} \frac{\partial V}{\partial x}\left(S_{s}\right)-\left(r+\frac{1}{\kappa(s)}\right) V\left(S_{s}\right)\right) d s+\frac{\kappa^{\prime}(s) s}{\kappa^{2}(s)} V\left(S_{s}\right) d s\right\} \\
& +e^{-\left(r+\frac{1}{\kappa(s)}\right) s} \frac{\partial V}{\partial x}\left(S_{s}\right) \sigma S_{s} d W_{s}
\end{aligned}
$$

Since for any positive $x, \frac{\sigma^{2} x^{2}}{2} \frac{\partial^{2} V}{\partial x^{2}}(x)+r x \frac{\partial V}{\partial x}(x)-\left(r+\frac{1}{\kappa(s)}\right) V(x) \leq 0, \kappa^{\prime}(s) \leq 0$, and $V(x) \geq 0$, the coefficient in the $d s$ term above is negative or zero, and the supermartingale property of the process $e^{-\left(r+\frac{1}{\kappa(t)}\right) t} V\left(S_{t}\right)$ follows.

\section{References}

[1] G. Barone-Adesi and R. E. Whaley, "Efficient Analytic Approximation of American Option Values," The Journal of Finance, Vol. XLII, No. 2, June 1987.

[2] J.-P. Fouque and C.-H. Han, "A martingale control variate method for option pricing with stochastic volatility," ESAIM: Probability and Statistics, 11, 40-54, (2007).

[3] J.-P. Fouque, G. Papanicolaou, and R. Sircar, "Derivatives in Financial Markets with Stochastic Volatility," Cambridge University Press, 2000.

[4] J.P. Fouque, G. Papanicolaou and R. Sircar, "From the Implied Volatility Skew to a Robust Correction to Black-Scholes American Option Prices," International Journal of Theoretical and Applied Finance, Vol. 4, No. 4 (2001). 651-675

[5] J.-P. Fouque, G. Papanicolaou, R. Sircar, and K. Solna, "Multiscale Stochastic Volatility Asymptotics," SIAM Journal on Multiscale Modeling and Simulation 2(1), 2003 (22-42).

[6] P. Glasserman, "Monte Carlo Methods in Financial Engineering," Springer Verlag, 2003.

[7] M. B. Haugh and L. Kogan "Pricing American Options: A Duality Approach," Operations Research, Vol. 52, No. 2, 258-270, 2004.

[8] F. Longstaff and E. Schwartz, "Valuing American Options by Simulation: A Simple Least-Squares Approach," Review of Financial Studies 14: 113-147, 2001.

[9] I. Karatzas, S. E. Shreve, "Brownian Motion and Stochastic Calculus," 2/e, Springer, 2000.

[10] L.C.G. Rogers, "Monte Carlo valuation of American Options," Math. Finance, 12, 271-286, 2002.

[11] J. Tsitsiklis and B. Van Roy, "Regression Methods for Pricing Complex American-style Options," IEEE Trans. Neural Networks, 12, 694-703, 2001. 